\title{
The Effect of Circumstellar Material on the Light Curves of Eclipsing Binary Systems
}

\author{
S. M. R. Ghoreyshi ${ }^{\mathrm{A}, \mathrm{B}, \mathrm{D}}$, J. Ghanbari $^{\mathrm{A}, \mathrm{C}}$, and F. Salehi ${ }^{\mathrm{C}}$ \\ ${ }^{A}$ Department of Physics, School of Sciences, Ferdowsi University of Mashhad, Mashhad, Iran \\ ${ }^{\mathrm{B}}$ Centro de Radioastronomia y Astrofisica, Universidad Nacional Autonoma de Mexico, \\ Apdo. Postal 72-3 (Xangari), Morelia, Michoacan 58089, Mexico \\ ${ }^{\mathrm{C}}$ Department of Physics, Khayyam Institute of Higher Education, Mashhad, Iran \\ D Corresponding author. Email: m.ghoreyshi@crya.unam.mx
}

\begin{abstract}
This study inspects the influence of various effects and free parameters of the accretion disc and circumstellar material on the emerging light curve of eclipsing binary systems that have a circumstellar disc, by using the SHELLSPEC code. The results indicate that some of the parameters, namely the temperature and inclination of the disc, spot, jet, stream and shell, significantly affect on the emerging light curve, while some other parameters, namely the exponent of the power-law behavior of the density of the disc, microturbulence, inner and outer radius of the disc, do not noticeably affect on the emerging light curve. An application to the Algol-type eclipsing binary system AV Del and an accretion disc model for the system using the SHELLSPEC code is included.
\end{abstract}

Keywords: variable stars — binaries — eclipsing binary — accretion disc — stars: individual (AV Del)

Received 2010 November 9, accepted 2011 August 18, published online 2011 October 4

\section{Introduction}

In recent years the accretion disc has been studied extensively. There are many astrophysical systems in the structure and evolution of which the accretion process has an important role. Accretion discs are seen in active galactic nuclei (AGNs), protoplanet clouds and binary systems. The accretion process usually occurs in a binary system when one of the companions fills its Roche lobe. Another companion can be a black hole, a neutron star, a white dwarf or a typical massive star. One type of binary system in which mass transfers are often currently seen between companions is the Algol-type binary system. The formation and properties of the accretion disc and circumstellar material in Algol-type systems are not very well understood.

Various complicated computer codes are presented for calculating and reversing light curves or spectra of binary stars with various shapes or geometry including the Roche model (Lucy 1968; Wilson \& Devinney 1971; Mochnacki \& Doughty 1972; Rucinski 1973; Hill 1979; Zhang et al. 1986; Djurasevic 1992; Drechsel et al. 1994; Vinkó et al. 1996; Hadrava 1997; Bradstreet \& Steelman 2002; Pribulla 2004). In the codes, the stars are assumed to be nontransparent and stripped of any circumstellar matter. An interesting approach for studying extended semitransparent atmospheres of some eclipsing binary components was developed by Cherepashchuk et al. (1984). Often the three-dimensional model of the circumstellar matter (behavior of state quantities and velocity field) is known or expected as a result of hydrodynamic simulations or observational constraints (see, e.g., Karetnikov et al. 1995; Richards \& Ratliff 1998). Unfortunately, threedimensional non-local thermodynamic equilibrium (NLTE) calculations and spectral synthesis including complex hydrodynamics are difficult to carry out, so one alternative has been to perform a simple volume integration of emissivity, which is often too simplified for the particular problem. On the other hand, highly sophisticated model atmospheres and spectrum synthesis codes have been developed assuming NLTE and planeparallel atmospheres of hot stars (Hubeny 1988; Hubeny \& Lanz 1992, 1995; Hubeny et al. 1994), spherically symmetric atmospheres (Kubát 2001), or stellar winds (Krtička \& Kubát 2002). There are also sophisticated stationary plane-parallel line-blanketed model atmospheres and spectrum synthesis codes for a large variety of stars assuming local thermodynamic equilibrium (LTE) (Kurucz 1993a, 1993b; Smith \& Dworetsky 1988; Piskunov 1992; and many others). However, these are very specialized codes and their main purpose is to calculate the spectrum emerging from a stellar atmosphere. It is difficult to apply them to the various cases outlined above. An exception is the special case of circumstellar matter in the form of accretion discs in cataclysmic variables (CVs). In such a case, the disc is approximated by a set of geometrically thin but optically thick static local atmospheres, and the output radiation is a sum of properly Doppler-shifted local 
emerging intensities (Orosz \& Wade 2003; Wade \& Hubeny 1998; la Dous 1989). However, in the case of optically thin discs or accretion disc winds, the Sobolev approximation is used (Progra et al. 2002; Long \& Knigge 2002; Rybicki \& Hummer 1983). Linnell \& Hubeny (1996) developed a package of codes that can calculate light curves or spectra of interacting binary stars including an optically thick (nontransparent) disc. However, it is often necessary to solve the radiative transfer in a moving disc at least along the line of sight, as demonstrated by Horne \& Marsh (1986). Nevertheless, their calculations assumed a linear shear and neglected stimulated emission, continuum opacity, and scattering, as well as a central star.

Budaj et al. (2005) attempted to bridge the gap in the previously mentioned approaches by using their code SHELLSPEC. It is a tool that solves in LTE the simple radiative transfer along the line of sight in an optional optically thin three-dimensional moving medium. Optional transparent (or nontransparent) objects such as a spot, disc, stream, jet, shell, or stars, as well as an empty space, may be defined (or embedded) in three dimensions and their composite synthetic spectrum calculated. The stars may have the Roche geometry and known intrinsic spectra. The code is quite a multipurpose, independent, and flexible tool that can also calculate a light curve or a trailing spectrogram when necessary, or it can be used to study various objects or effects. Also, they performed investigations in order to study the underlying physics of the structures of Algol-type eclipsing binaries, by using the SHELLSPEC code. They calculated synthetic $\mathrm{H} \alpha$ spectra of TT Hya, which is an Algol-type eclipsing binary with an accretion disc. They studied the influence of various effects and free parameters of the disc on the emerging spectra. Also, Ghoreyshi et al. (2011) used the SHELLSPEC code to solve the light curve of the Algol-type eclipsing binary system AV Del. But a complete study of the effect of these parameters on the light curve is not published.

AV Del $\left(\alpha_{2000}=20^{h} 45^{m} 31^{s} .47, \delta_{2000}=11^{\circ} 10^{\prime} 26^{\prime \prime} .4\right.$, $V=11.8)$ is a rather neglected variable star which was discovered by Hoffmeister (1935) and given the original designation 184.1930. It is a suspected member of the cool Algols (Popper, 1996) and was reported by the author as a double-lined system with a period of 3.85 days and a mean spectral type of G5, although other sources have classified it as F8 (e.g., Halbedel 1984). The rare class of cool Algol systems are distinguished from the classical Algol systems in that the mass-gaining component is a late-type star rather than a B- or A-type star. Mader et al. (2005) presented new spectroscopic and BVRI photometric observations of AV Del. They determined the radial velocities for both components, using the twodimensional cross-correlation technique TODCOR (Zucker \& Mazeh 1994). To present a detailed radial velocity and light curve analysis of the optical data, Mader et al. (2005) combined all light curve data with the spectroscopic observations and analyzed them using the Wilson-Devinney (WD) code (Wilson \& Devinney
1971; Wilson 1979, 1990). They showed the system to be most likely semi-detached, with the less massive and cooler star filling its Roche lobe.

This paper tries to explore in more detail the effects of various input parameters on the light curve of a typical Algol-type eclipsing binary system, by using the SHELLSPEC code (Section 2).

In Section 3 we explain the simulation results. At first we discuss the effects of various input parameters on the light curve of a typical Algol-type eclipsing binary system, and then show how the SHELLSPEC code describes the light curve of the Algol-type eclipsing binary system AV Del and summarize the results in Section 4.

\section{Code Description}

When a star (a member of a binary) in a circular orbit expands to fill and then overfill its Roche lobe, it will start to lose mass to its companion. Matter that leaves the surface of one component of a binary star can be partly or wholly accreted by the companion. The matter can directly strike the gainer, or it can be stored in an accretion disc around it, and part of the matter may leave the system.

In a common situation, the matter that feeds the disc flows from the companion star through the inner Lagrangian point that connects the two Roche lobes in the binary system. The matter thus leaves the mass -losing star in a rather thin stream in the orbital plane of the two stars. In reality, the matter may spray in a messier fashion, but most of the matter remains in the orbital plane. If the two stars were stationary, this matter would flow from one star to the other directly along the line connecting the centers of the two stars and strike the mass-gaining star. In a binary star system, however, the stars are constantly moving in orbit, so the mass-gaining star is a moving target. The matter may leave the mass-losing star headed for the other star, but because the other star moves along in its orbit, the transferred matter cannot fall directly onto the mass-gaining star.

The gravitational domain of the mass-gaining star captures the matter, however, so the stream circles around and collides with the incoming stream. As this process continues, the flow of self-interacting matter will form first a ring and then a disc (Wheeler, 2007).

Accretion discs often show P-Cyg type absorption lines that may be caused by coronal heating above and below the disc. A tenuous wind may be driven away from the disc, somewhat like a stellar wind (Eggleton, 2006). Moreover, a thin shell of gas may surround the primary star that usually expands because of the stellar wind of the primary.

The interaction of the mass stream with the disc (in the place where the gas stream from the secondary strikes the edge of the disc) causes a bright hot spot which is seen as additional light in the light curve.

Many objects that involve accretion discs are also seen to be accompanied by bipolar jets apparently emerging from the central region normal to the disc. Jets are also 
seen in active galactic nuclei (AGN), where it is believed that a central massive black hole is accreting neighboring material.

The appearance to an observer varies greatly depending on the observer's location relative to the jet direction. Other stellar objects also exhibit accretion discs and jets, such as protostars and $\mathrm{X}$ ray-emitting black hole binary systems. Collimated polar jets, taking angular momentum away from the disc, allow most disc matter to fall onto the compact object.

Protostars are embryonic stars in their formative stages before setting on the main sequence. Black hole binaries are sometimes called microquasars because they exhibit relativistic jets similar to those of extragalactic quasars. In the binary accreting systems, the accreted matter is provided by a normal gaseous companion star. The masses of the black hole in microquasars are thought to be of order $10 \mathrm{M}_{\odot}$ (Bradt, 2008).

All such effects can play an important role in the evolution of binary systems. Therefore, studying and deliberating on the existence and the structure of these effects can give us insights into the interacting binary systems.

The Algol-type binaries are interacting systems consisting of a hot, usually more massive, main-sequence star and a cool giant or subgiant that fills its Roche lobe. The secondary is losing the mass to its companion, and we are observing the circumstellar material in the form of a gas stream, a jet, a shell, an accretion annulus, or an accretion disc. The most recent paper that summarizes our current knowledge of the Algols is the work by Richards \& Albright (1999).

We have used the SHELLSPEC code that is to our knowledge the only tool that can produce the synthetic light curves of interacting binaries, taking into account optically thin moving circumstellar matter. The code was applied to the typical Algol-type eclipsing binary which has an accretion disc. Synthetic light curves of the system were calculated by varying the parameters.

The SHELLSPEC code is designed to solve simple radiative transfer along the line of sight in three-dimensional moving media. The scattered light from a central object is taken into account assuming an optically thin environment (Budaj \& Richards 2004). Output intensities are then integrated through the two-dimensional projection surface of a three-dimensional object. The assumptions of the code include LTE and optical known-state quantities and velocity fields in three dimensions (Budaj \& Richards 2004). In the code, both objects, the primary and the companion (secondary), may be shaped according to the Roche model used for detached, semi-detached or contact systems.

Light curve changes were studied considering variation of these parameters: geometrical shape of the disc, half thickness of the disc cylinder, the exponent of the power-law behavior of the densities $\rho \sim r^{\eta}$ in the disc, effective temperature of the disc, inclination of the disc, microturbulence, inner radius of the disc, outer radius

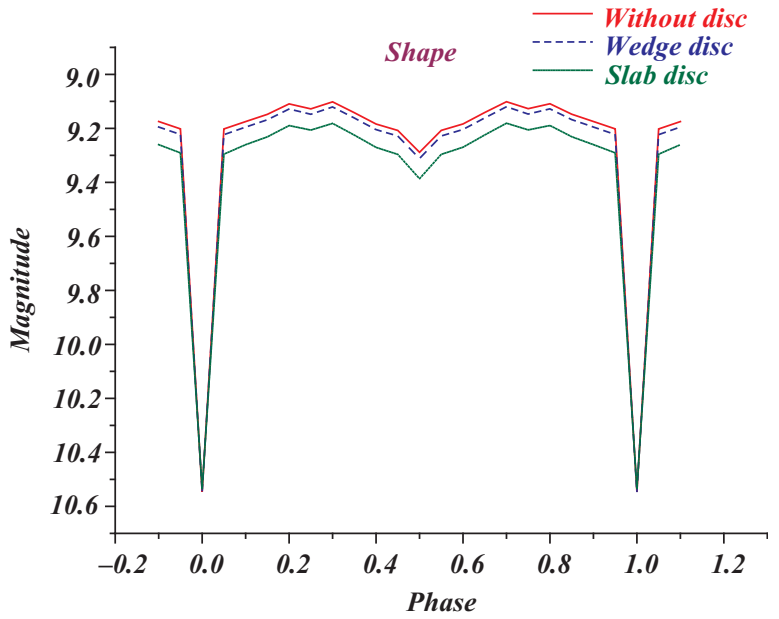

Figure 1 Effect of the geometrical shape of the disc on the emerging light curve of a typical binary system.

of the disc, and also the existance of spots, jets, streams and shells.

\section{Changes to Light Curve from Varying Model Parameters}

Now, the purpose is to study in more detail the influence of the effects on the light curve of a typical Algol-type eclipsing binary system. At first, a typical Algol-type eclipsing binary was considered in which the primary star was surrounded by an accretion disc. The primary was treated as a rotating solid sphere while the surface of the secondary was modeled in terms of the Roche model. The primary has the following characteristics: the mean temperature $T_{1}=9800 \mathrm{~K}$, the mass $M_{1}=2.6 \mathrm{M}_{\odot}$ and the radius $R_{1}=1.9 \mathrm{R}_{\odot}$. Also, the secondary has the following characteristics: the mean temperature $T_{2}=4600 \mathrm{~K}$, the mass $M_{2}=0.6 \mathrm{M}_{\odot}$ and the radius $R_{2}=5.9 \mathrm{R}_{\odot}$. The considered disc is an optically thick Keplerian disc that has the following characteristics: inner radius $R_{\text {in }}=$ $2.0 \mathrm{R}_{\odot}$, outer radius $R_{\text {out }}=9.0 \mathrm{R}_{\odot}$, effective temperature $T_{\text {disc }}=7000 \mathrm{~K}$ and half of the thickness of the disc cylinder $\alpha=1.0 \mathrm{R} \odot$. The electron number density of the disc was set to $n_{e}=2 \times 10^{10} \mathrm{~cm}^{-3}$, density $\rho=3.3 \times$ $10^{-14} \mathrm{~g} \mathrm{~cm}^{-3}$, microturbulence $v_{\text {trb }}=30 \mathrm{~km} \mathrm{~s}^{-1}$ and inclination $i=82^{\circ} .84$.

The parameters were allowed to vary one by one and the others were kept fixed. Therefore, our calculations illustrate the effect of varying just one free parameter at a time while keeping all the others fixed. In many cases this may not represent the real behavior of the system, since some quantities may be more or less interlocked, such as temperatures and electron number densities. Nevertheless, the study give us a general idea of the behavior of the system when the free parameters vary.

Figure 1 displays the effect of the geometrical shape of the disc. Here, three profiles are compared: undermost profile was obtained for the slab-shaped disc with the parameters listed earlier. The middle profile was obtained 

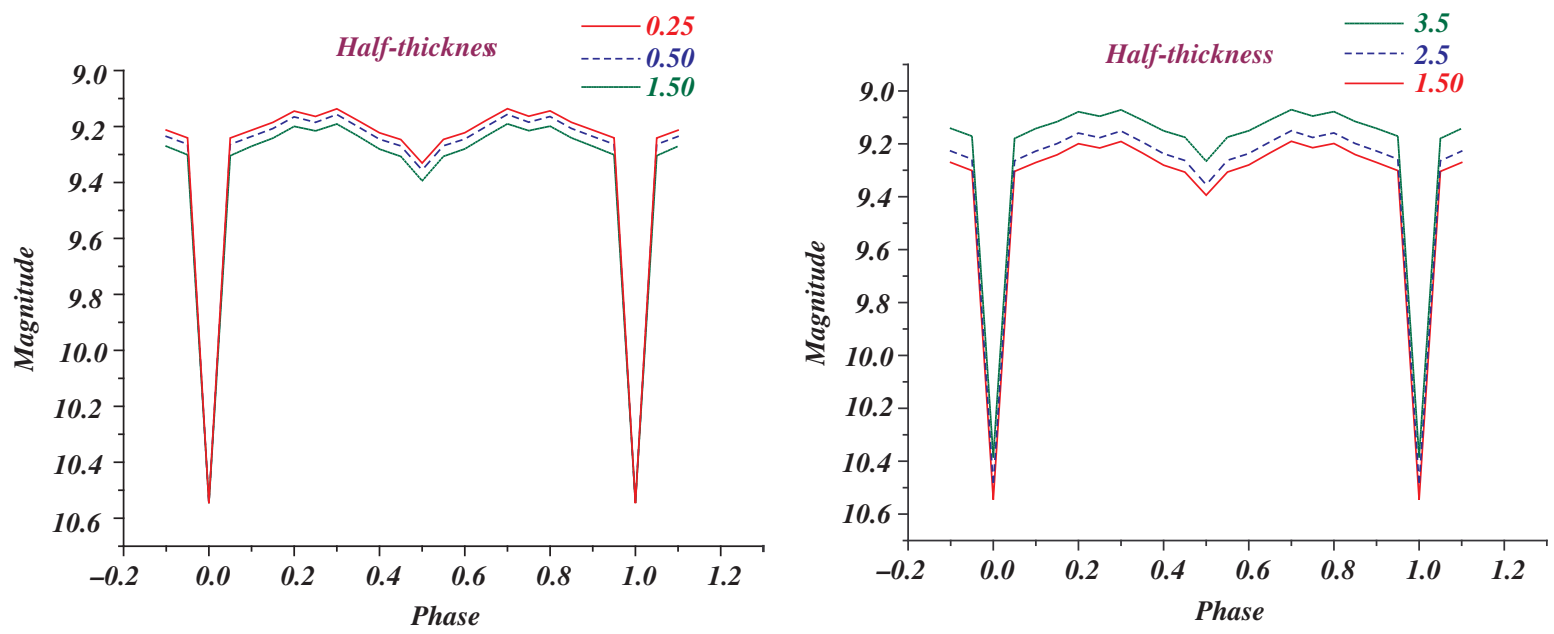

Figure 2 Left: Effect of varying $\alpha$ (vertical half-width of the disc) from 0.25 to $1.5 \mathrm{R}_{\odot}$; right: effect of varying vertical half-width of the disc from 1.5 to $3.5 \mathrm{R}_{\odot}$.

for the shape of a rotating wedge with the wedge angle $\alpha=25^{\circ}$. It is clear that both profiles are similar, except that the rotating wedge shape produces a stronger sytem luminosity than the slab disc model because the rotating wedge-shaped disc has a larger Keplerian emission surface than the slab disc model. The upper profile was obtained for the case without a disc. As the considered disc has a lower effective temperature than the primary, the overall luminosity increases when there is no disc. Of course if the inclination decreases, the emission surface of the disc increases and the overall luminosity of the system increases. Also, it is clear that the main difference between the three profiles occurs away from the primary eclipse.

Figure 2 clarifies the effect of increasing the vertical half-thickness of the disc from 0.25 to $3.5 \mathrm{R}_{\odot}$ for the slabshaped disc model. The luminosity is weakest at about $1.5 \mathrm{R}_{\odot}$. The luminosity of the system decreases from 0.25 to $1.5 \mathrm{R}_{\odot}$ since the accretion disc, which has a lower temperature than the primary star, creates an eclipse (similar to the secondary). Increasing the vertical halfthickness from 1.5 to $3.5 \mathrm{R}_{\odot}$ increases the luminosity of the system since increasing the emitting space volume dominates the effect of the eclipse. It is clear that the main difference between the profiles is out of the primary eclipse.

Figure 3 indicates the effect of increasing the vertical half-thickness of the disc from $10^{\circ}$ to $30^{\circ}$ for the rotating wedge disc model. The luminosity of the system increases as the emitting space volume increases. The wedge angle $\alpha=25^{\circ}$ was considered, therefore the half-thickness of the disc near the surface of the primary is about $0.9 \mathrm{R}_{\odot}$ and the half-thickness of the disc at the outer limb of the disc is about $4 \mathrm{R}_{\odot}$. Thus, similar to the slab disc model, in the current shape increases in the emitting space volume dominate the effect of the eclipse by the disc.

Figure 4 (top left and top right) displays the effect of changing the outer radius of the disc from 5 to $11 \mathrm{R}_{\odot}$. For $R_{\text {out }}=7 \mathrm{R}_{\odot}$, the luminosity of the system has the

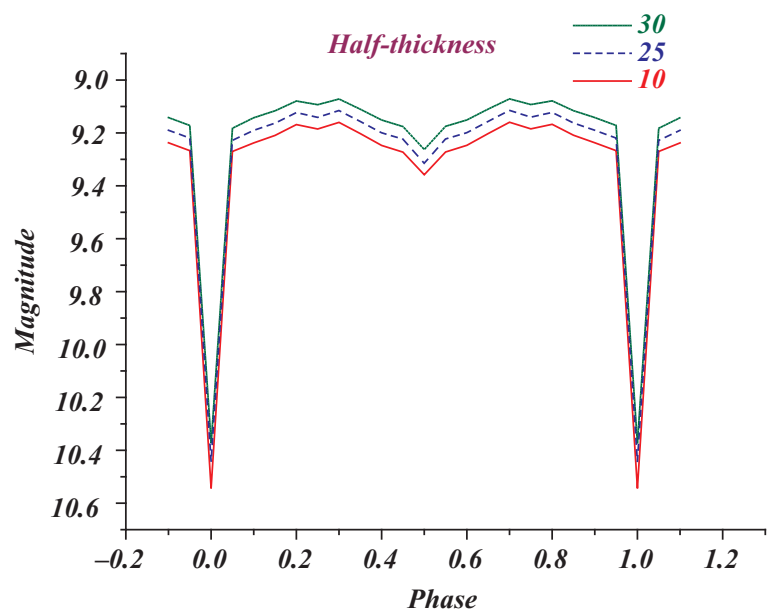

Figure 3 Effect of varying the vertical half-width of the disc from $10^{\circ}$ to $30^{\circ}$ for the rotating wedge disc model.

minimum amount. The luminosity of the system decreases from 5 to $7 \mathrm{R}_{\odot}$, and increases from 7 to $11 \mathrm{R}_{\odot}$. The evidence of this showing is similar to the case of the vertical half-thickness parameter. Increasing the outer radius from 5 to $7 \mathrm{R}_{\odot}$, the disc plays an obstructive role between us and the light of the primary star. Then, increasing toward higher radius, the influence of the increase in the emitting space volume overcomes the influence of eclipse, so the overall luminosity increases. Nevertheless, the difference between the profiles for 5 to $11 \mathrm{R}_{\odot}$ is not very noticeable, especially for 5 to $7 \mathrm{R}_{\odot}$.

The bottom part of Figure 4 displays the effect of increasing the inner radius of the disc from 2 to $8 \mathrm{R}_{\odot}$. Increasing from 2 to $3 \mathrm{R}_{\odot}$, the luminosity decreases, since the emitting space volume decreases. Increasing from 3 to $8 \mathrm{R}_{\odot}$, the luminosity increases since a smaller volume of the disc lies between the primary star and us. For $R_{\text {in }}=8 \mathrm{R}_{\odot}$ we have an accretion annulus instead of the accretion disc. Here again the difference between the 

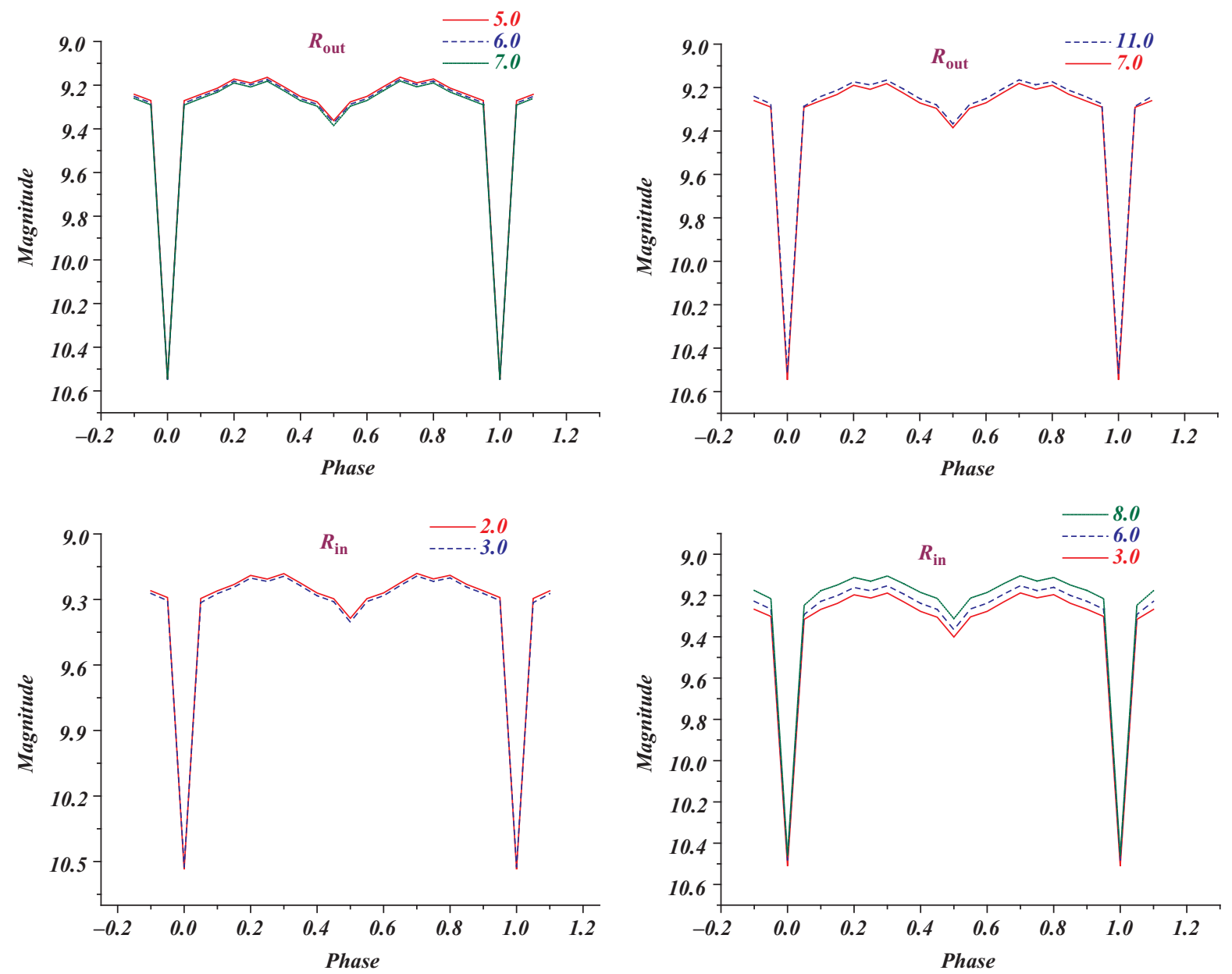

Figure 4 Top left: Effect of varying the outer radius of the disc from 5 to $7 \mathrm{R}_{\odot}$; top right: effect of varying the outer radius of the disc from 7 to $11 \mathrm{R}_{\odot}$; bottom left: effect of varying the inner radius of the disc from 2 to $3 \mathrm{R}_{\odot}$; bottom right: effect of varying the inner radius of the disc from 3 to $8 \mathrm{R}_{\odot}$.

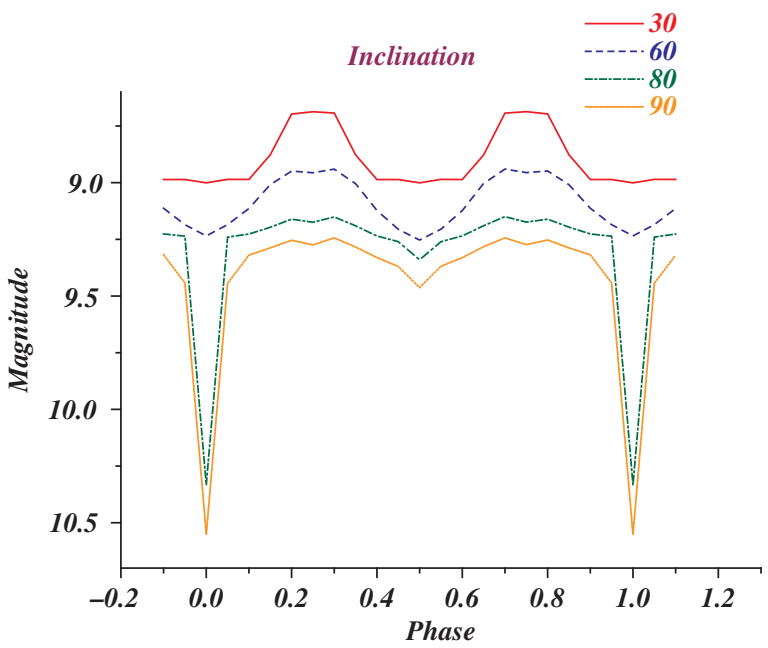

Figure 5 Effect of changing the inclination of the disc from $90^{\circ}$ to $30^{\circ}$.

profiles is not very noticeable and is out of the primary eclipse.

Figure 5 illustrates the light curve if the disc were viewed from different inclination angles. Starting from edge-on, $i=90^{\circ}$, the system has the least luminosity and the deepest primary eclipse. As the inclination decreases and one begins to see the disc pole-on, the luminosity increases and the shape of the light curve noticeably changes since that part of the surface of the system facing toward us increases. At the inclination angle $i=60^{\circ}$ the depths of the primary and secondary eclipses are comparable. In fact, the depth of the primary eclipse greatly decreases and the depth of the secondary eclipse slightly increases. At the inclination angle $i=30^{\circ}$, the depth of the primary and secondary eclipses are approximately equal and their expanses greatly increase.

Figure 6 displays the effect of varying the temperature of the disc over the interval $5000-12000 \mathrm{~K}$. The luminosity is the weakest at about $6000 \mathrm{~K}$ and increases toward higher temperatures, since the temperature of the disc will be comparable with that of the primary. It is clear that increasing the temperature does not affect the shape of the light curve but increases or decreases the luminosity out of the primary eclipse.

If the density and electron number density be inhomogeneous and have a power-law dependence on the distance from the star, namely, $\rho \sim r^{\eta}$, Figure 7 clarifies 

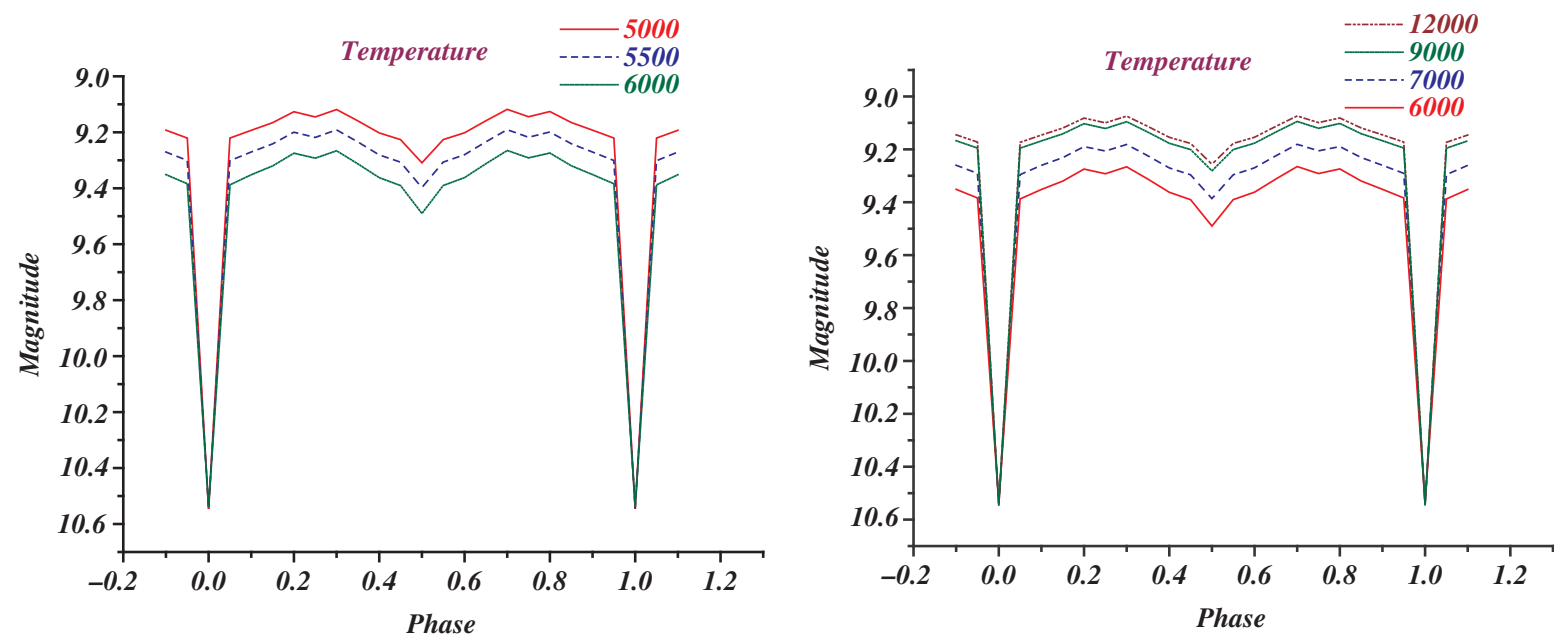

Figure 6 Left: Effect of varying temperature of the disc from 5000 to $6000 \mathrm{~K}$; right: effect of varying temperature of the disc from 6000 to $12000 \mathrm{~K}$.

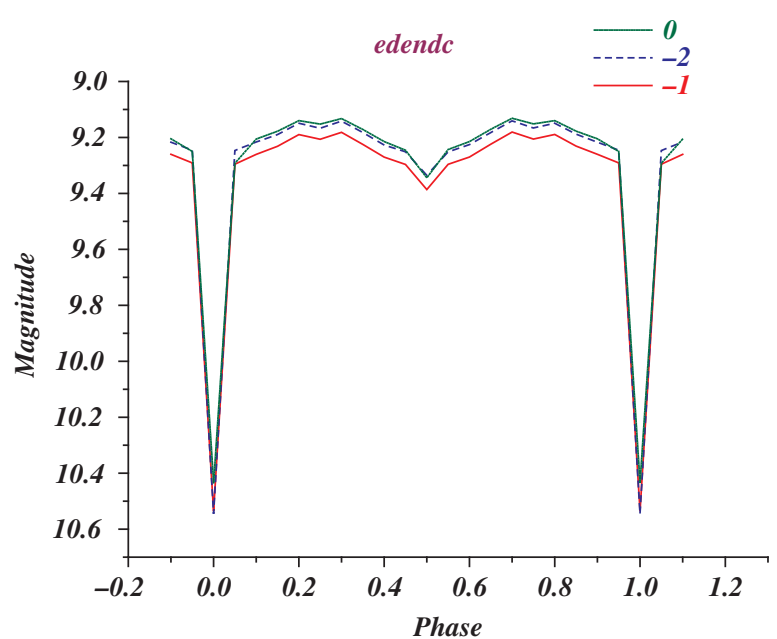

Figure 7 Effect of varying the exponent $\eta$ of the power-law behavior of the densities $\rho \sim r^{\eta}$ in the disc from 0 to -2 .

the effect of varying the exponent $\eta$ from 0 to -2 . The parameter noticeably affects the emerging spectrum (Budaj et al. 2005) but the light curve is not so sensitive to the density. It is obvious from the figure that the difference between profiles for $\eta=0$ and $\eta=-2$ is not noticeable but for $\eta=-1$ there is a greater difference from $\eta=0$ and $\eta=-2$ and the luminosity for $\eta=0$ and $\eta=-2$ is greater than for $\eta=-1$.

From comparing densities for $\eta=-1$ and $\eta=-2$, we find that the density at the outer region of the disc is less than its inner region so that it tends to be less if it is considered for $\eta=-2$. Therefore we expect more luminosity for a binary system in the latter case than the former. Since density is less, its causes less effect on the emerging light of the star via attracting or scattering. Of course, we must notice that the inclination of disc can influence the emission surface of the disc and so change the total luminosity of system. Also, the density is fixed and independent of $r$ for $\eta=0$. In this case, we receive

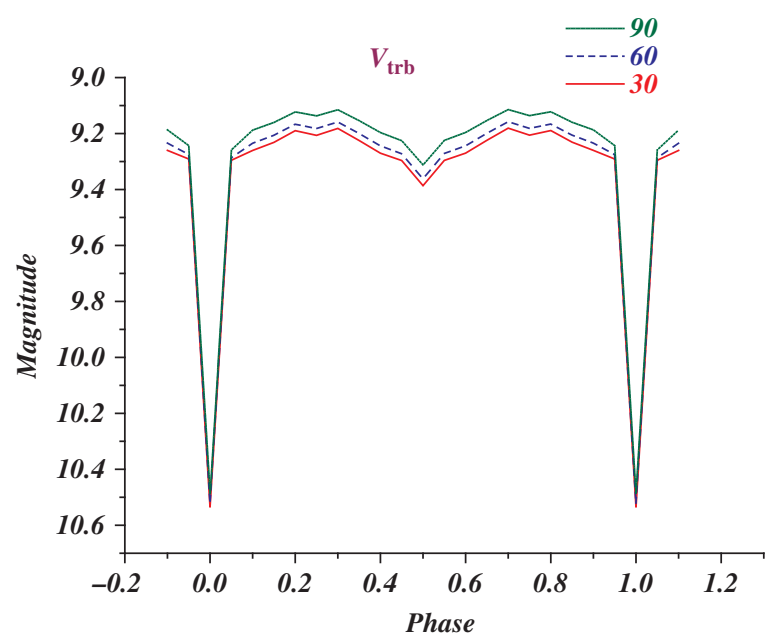

Figure 8 Effect of increasing the microturbulence in the disc from 30 to $90 \mathrm{~km} \mathrm{~s}^{-1}$.

more luminosity for the binary system than in the former case. It has the same role in attracting or scattering of the elements of the disc. In the results, the change in total luminosity isn't very noticeable.

Figure 8 shows the effect of increasing the microturbulence of the disc from 30 to $90 \mathrm{~km} \mathrm{~s}^{-1}$. Increasing the microturbulence of the disc, the overall luminosity of the system increases, too. As in most of earlier cases, the changes in the profile are seen out of the primary eclipse.

Figure 9 (top left) illustrates the influence of the existence of a spot on the light curve. It is clear that the spot can noticeably affect the luminosity of the system. The main difference is in the primary eclipse. In the figure a spot was considered which has the effective temperature $T_{\text {spot }}=4400 \mathrm{~K}$ and radius $R_{\text {spot }}=1 \mathrm{R} \odot$. Therefore, the spot causes an increase in the luminosity of the system. The amount of difference between profiles depends on the free parameters of the spot. 

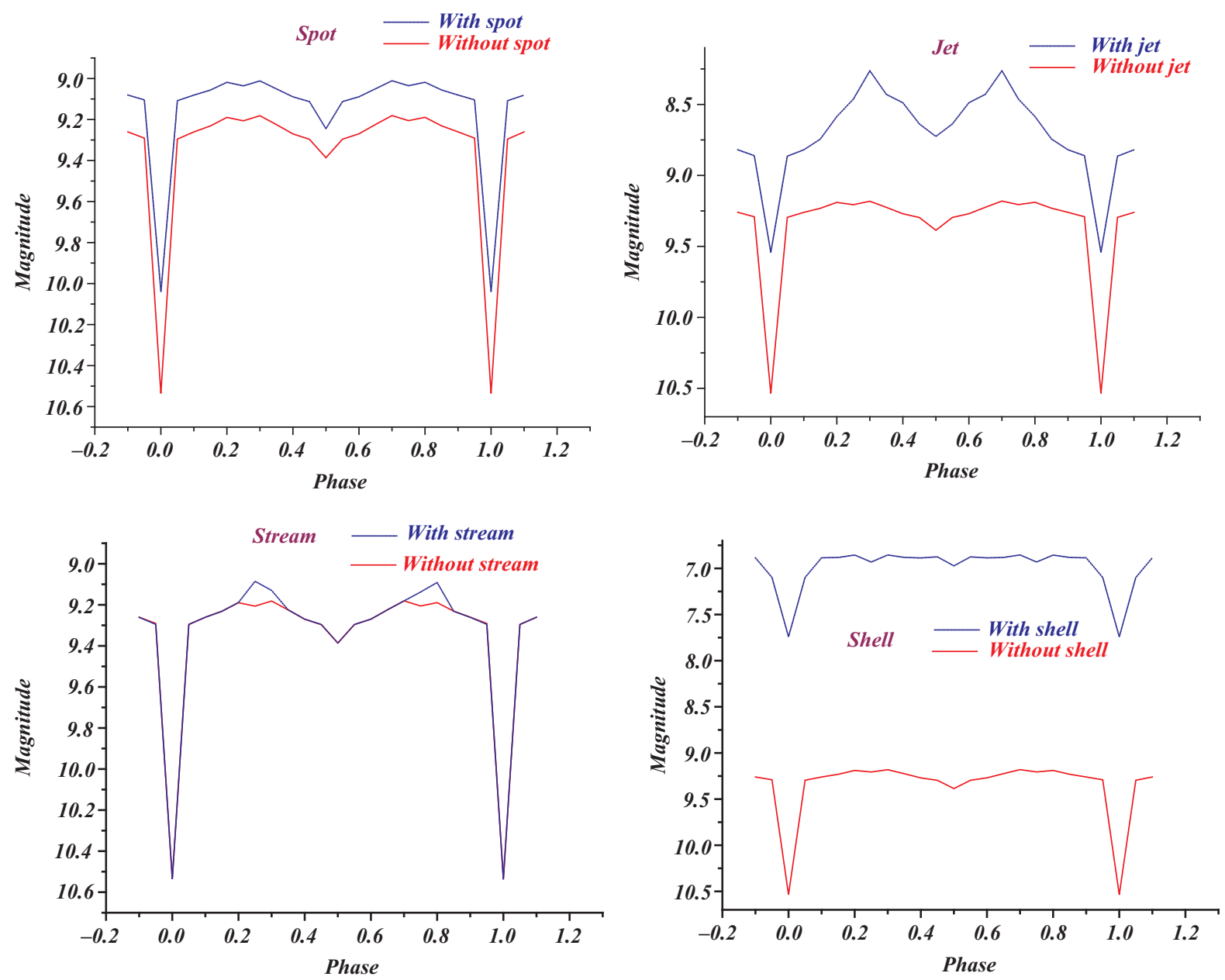

Figure 9 Top left: Effect of existence of a spot on the emerging light curve of a typical binary system; top right: effect of existence of a jet on the emerging light curve of a typical binary system; bottom left: effect of existence of a stream on the emerging light curve of a typical binary system; bottom right: effect of existence of a shell on the emerging light curve of a typical binary system.

Figure 9 (top right) displays the influence of the existence of a jet on the light curve. It was considered that the jet has the following characteristics: angle halfwidth of the jet cones $\theta=10^{\circ}$, inner radius boundaries of the jet cones $r_{\text {in }}=1.0 \mathrm{R}_{\odot}$, outer radius boundaries of the jet cones $r_{\text {out }}=5.0 \mathrm{R}_{\odot}$ and radial (expanding) velocity of the jet $V_{\mathrm{j}}=360 \mathrm{~km} \mathrm{~s}^{-1}$. As it is shown in Figure 5, the jet noticeably changes the shape of the light curve and increases the overall luminosity of the system. Existence of the jet causes a decrease in the depth of the primary eclipse and and an increase in the depth of the secondary eclipse. Also, the shape of the light curve changes the interval phases 0.1 to 0.4 and 0.6 to 0.9 . In the intervals two wings appear. At the primary eclipse the jet cannot be seen completely because the secondary star covers a part of the jet, but at the interval phases 0.1 to 0.4 and 0.6 to 0.9 the jet can be seen faintly. Also, the secondary star is not in eclipse, therefore the two wings appear. Possibly, it is not suitable that the effect of the existence of the jet in the Algol-type binary systems was considered since usually one cannot see the jet in these systems. Perhaps it would be better to investigate the influence of the jet in binary systems which include white dwarfs, neutron stars or black holes, but the aim of this study was to display the effect of the jet on the shape of the light curve of Algoltype systems, even if it is not so realistic.

Figure 9 (bottom left) illustrates the effect of the existence of the stream on the light curve. The considered stream has the following characteristics: stream velocity at the beginning of the stream $V_{1}=250 \mathrm{~km} \mathrm{~s}^{-1}$, stream velocity at the end of the stream $V_{2}=530 \mathrm{~km} \mathrm{~s}^{-1}$ and radius of the stream $r_{\mathrm{s}}=1 \mathrm{R}_{\odot}$. It is clear that the stream did not change the depth or the expanse of the primary and secondary eclipses and did not change the luminosity of the system, but it causes the appearance of two wings in the first and second quadrature of the light curve.

Figure 9 (bottom right) displays the effect of the existence of a shell around the system. The considered shell has the following characteristics: velocity of the uniformly expanding shell $V_{\mathrm{sh}}=40 \mathrm{~km} \mathrm{~s}^{-1}$ and mean temperature of the shell $T_{\mathrm{sh}}=9500 \mathrm{~K}$. It is clear that the shell greatly increases the luminosity of the system. Also, the shell causes a decrease in the depth of the primary eclipse.

The purpose of the paper was not to study the effect of the free parameters of the spot, jet, stream and shell on the 


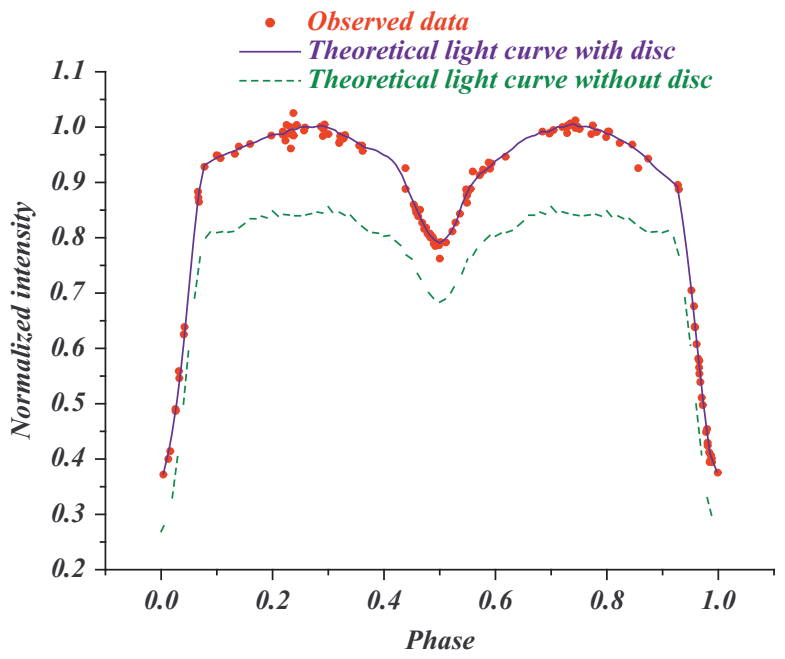

Figure 10 The observed and the theoretical light curves of AV Del. The theoretical light curves are drawn on the basis of the values derived via SHELLSPEC code. The observed data are shown by solid circles and the theoretical light curves are shown by continuous (with disc) and dashed (without disc) lines.

light curve of the system. Nevertheless, the effect of the existence of these objects on the light curve of the system was studied.

Now that we understand the influence of various parameters on the emerging light curve, we can proceed to fit the real light curve of AV Del. We used the photometric data reported by Mader et al. (2005) for analysis via the SHELLSPEC code. A detailed study of these data is published in Ghoreyshi et al. (2011), in which an analysis of the photometric and radial-velocity data of the system by using both Wilson-Devinney code (Wilson \& Devinney 1971; Wilson 1979, 1990) and the SHELLSPEC code performed.

We performed some sophisticated calculations of the light curve in which both stars and a disc were considered. The primary was treated as a rotating solid sphere, while the surface of the secondary was modeled in terms of the Roche model. First, the code was run assuming there was no third body but it failed to obtain a good fit to the observed data. Then a disc only was included as a third body. On the basis of the configuration, agreement between the observed and the theoretical light curve was very good, although the paper tried to examine the effect of other bodies (spot, jet, stream and shell) on the light curve. Finally, the best fit was obtained while only an accretion disc was considered. As we see in Figure 10, when the disc is included, the agreement between the observed data and the theoretical light curve is quite good. It should be mentioned that the solution derived in this way is not a unique one. Of course, it shows that the fit is remarkably improved by assuming a disc of some reliable dimensions and physical parameters.

The parameters of the disc are given in Table 1. As we see, the shape of the disc was found to be a slab cylinder with the half-thickness (adisc) $1.2 \mathrm{R}_{\odot}$. The inner and outer radius of the disc (rindc and routdc, respectively)
Table 1. The parameters of the accretion disc of AV Del

\begin{tabular}{ll}
\hline Parameter & Value \\
\hline adisc $\left(\mathrm{R}_{\odot}\right)$ & 1.2 \\
rindc $\left(\mathrm{R}_{\odot}\right)$ & 3.5 \\
routdc $\left(\mathrm{R}_{\odot}\right)$ & 8.0 \\
tempdc $(\mathrm{K})$ & 5700 \\
densdc $\left(\mathrm{g} \mathrm{cm}^{-3}\right)$ & $33 \times 10^{-15}$ \\
anedc $\left(\mathrm{cm}^{-3}\right)$ & $21 \times 10^{9}$ \\
edendc & -1 \\
vtrbdc $\left(\mathrm{km} \mathrm{s}^{-1}\right)$ & 90 \\
\hline
\end{tabular}

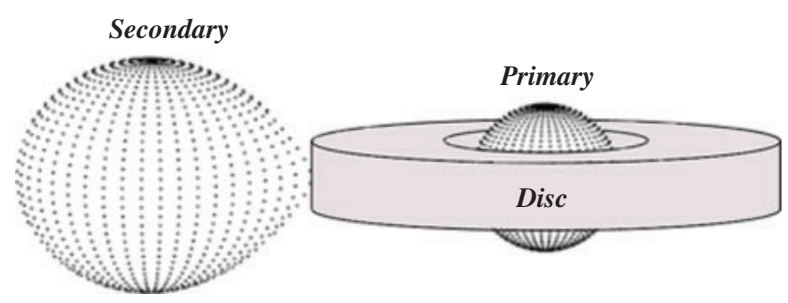

Figure 11 The configuration of the binary system AV Del on the basis of the values derived using the SHELLSPEC code. As we see, the primary star is surrounded by an accretion disc.

were obtained as 3.5 and $8.0 \mathrm{R}_{\odot}$. Therefore, the inner edge of the disc is $0.99 \mathrm{R}_{\odot}$ away from the surface of the primary star, since the radius of the primary star was obtained as $2.51 \mathrm{R}_{\odot}$ (Ghoreyshi et al. 2011). Moreover, on the basis of the values 4.25 and $13.32 \mathrm{R}_{\odot}$ for the radius of the secondary star and the distance between the center of the stars, respectively (Ghoreyshi et al. 2011), it is clear that the outer edge of the disc is $1.07 \mathrm{R}_{\odot}$ away from the surface of the secondary star.

The effective temperature of the disc (tempdc) was obtained as $5700 \mathrm{~K}$. It is close to that of the primary star $(6000 \mathrm{~K})$. Therefore, in this system, the disc is treated as a hot object (in comparison to the primary star) and increases the overall luminosity of the system. This is the reason that in Figure 10 we see the system with disc has more luminosity than that without disc. (This goes against what was said of Figure 1, since in the latter case the effective temperature of the primary star and disc were assumed to be 9800 and $7000 \mathrm{~K}$, respectively. So the disc cannot be a hot object. Also, the overall volume of the disc supposed in Figure 1 is bigger than that of this real case and with such temperature, the disc behaves as a cold object in the system and decreases its overall luminosity. All these points help to justify the difference between Figure 1 and Figure 10.)

The mean density of the disc (densdc) and the electron number density of the disc (anedc) were obtained as $33 \times 10^{-15} \mathrm{~g} \mathrm{~cm}^{-3}$ and $21 \times 10^{9} \mathrm{~cm}^{-3}$, respectively. Finally, the best values for the exponent of the powerlaw behavior of the densities $\rho \sim r^{\eta}$ in the disc (edendc) and microturbulence $\left(v_{\text {trb }}\right)$ were obtained for -1 and $90 \mathrm{~km} \mathrm{~s}^{-1}$, respectively.

The configuration of the system while its primary star is surrounded by the accretion disc on the basis of the values of Table 1 is shown in Figure 11. 


\section{Conclusion}

We have used a new computer code called SHELLSPEC for studying the effect of various free parameters of circumstellar material on the light curve of a typical Algol-type eclipsing binary system. It is the first time that the effect of such parameters are considered. The temperature and inclination of the disc have the strongest effect on the emerging light curve of the system, while the inner and outer radius of the disc have the weakest effect on the light curve. This is in agreement with what Budaj et al. (2005) derived about the spectrum of TT Hya.

The code was applied to the well-known eclipsing Algol-type binary system AV Del, which has an accretion disc. The light curve of the system for all phases were calculated by taking into account a spherical primary and a Roche lobe-filling secondary, as well as a disc. It was showed that without an accretion disc the light curve of the system cannot be described. Therefore, we believe this study can be a verification for the use of the SHELLSPEC code in analysis of eclipsing binary systems, especially for those who are doubtful about existing circumstellar material in their structure. Also, it can be suggested for future investigations of such systems that the SHELLSPEC code be used for analysis of the systems' light curves to consider whether they contain any kind of circumstellar material or not. Of course, as an important weakness for the SHELLSPEC code in comparison with other codes (such as the Wilson-Devinney code), we can mention the inability of the SHELLSPEC code to derive the uncertainty for the parameters so that one does not know how reliable the values obtained for the parameters are. Otherwise, the SHELLSPEC code really is a strong code for analysis of eclipsing binary systems.

\section{Acknowledgments}

We wish to thank Dr. Jano Budaj for his very useful comments at different stages of the work. Also we would like to thank the referee for his or her comments and suggestions.

\section{References}

Bradstreet, D. H. \& Steelman, D. P., 2002, BAAS, 34, 1224

Bradt, H., 2008, Astrophysics Processes, (Cambridge: Cambridge University Press)

Budaj, J. \& Richards, M. T., 2004, Contrib. Astron. Obs. Skalanté Pleso, 34, 167

Budaj, J., \&, Richards, M. T. \& Miller, B., 2005, ApJ, 623, 411

Cherepashchuk, A. M., Eaton, J. A. \& Khaliullin, K. F., 1984, ApJ, 281,774
Djurasevic, G., 1992, Ap\&SS, 197, 17

Drechsel, H., Haas, S., Lorenz, R. \& Mayer, P., 1994, A\&A, 284, 853

Eggleton P., 2006, Evolutionary Processes in Binary and Multiple Stars, (Cambridge: Cambridge University Press)

Ghoreyshi, S. M. R., Ghanbari, J. \& Salehi, F., 2011, PASA, 28, 1

Hadrava, P., 1997, A\&AS, 122, 581

Halbedel, E. M., 1984, IBVS, 2549, 1

Haffmeister, C., 1935, Astron. Nature, 255, 401

Hill, G., 1979, Publ. Dom. Astrophys. Obs. Victoria, 15, 297

Horne, K. \& Marsh, T. R., 1986, MNRAS, 218, 761

Hubeny, I., 1988, Comput. Phys. Commun, 52, 103

Hubeny, I. \& Lanz, T., 1992, A\&A, 262, 501 , 1995, ApJ, 439, 875

Hubeny, I., Lanz, T., \& Jeffery, C. S., 1994, in Newsletter on Analysis of Astronomical Spectra 20, ed. C. S. Jeffery (CCP7; St. Andrews: St. Andrews Univ.), 30

Karetnikov, V. G., Menchenkova, E. V. \& Nazarenko, V. V., 1985, AN, 316, 163

Krtička, J. \& Kubát, J., 2002, A\&A, 388, 531

Kubát, J., 2001, A\&A, 366, 210

Kurucz, R. L., 1993a, Kurucz CD-ROM 18, SYNTHE Spectrum Synthesis Programs and Line Data (Cambridge: SAO)

—., 1993b, Kurucz CD-ROM 13, ATLAS9 Stellar Atmosphere Programs and $2 \mathrm{~km} \mathrm{~s}^{-1}$ grid (Cambridge: SAO)

la Dous, C., 1989, A\&A, 211, 131

Linnell, A. P. \& Hubeny, I., 1996, ApJ, 471, 958

Long, K. S. \& Knigge, C., 2002, ApJ, 579, 725

Lucy, L. B., 1968, ApJ, 153, 877

Mader, J. A., Torres, G., Marshall, L. A. \& Rizvi, A., 2005, AJ, 130,234

Mochnacki, S. W. \& Doughty, N. A., 1972, MNRAS, 156, 51

Orosz, J. A. \& Wade, R. A., 2003, ApJ, 593, 1032

Piskunov N. E., 1992, in Stellar Magnetism, ed. Yu. V. Glagolevskij \& I. I. Romanyuk (Saint Petersburg: Nauka), 92

Popper, D. M., 1996, ApJS, 106, 133

Pribulla, T., 2004, in ASP Conf. Ser. 318, Spectroscopically and Spatially Resolving the Components of Close Binary Stars, ed. R. Hilditch et al. (San Francisco: ASP), 117

Progra, D., Kallman, T. R., Drew, J. E. \& Hartley, L. E., 2002, ApJ, 572,382

Richards, M. T. \& Albright, G. E., 1999, ApJS, 123, 537

Richards, M. T. \& Ratliff, M. A., 1998, ApJ, 493, 326

Rucinski, S. W., 1973, Acta. Astron, 23, 79

Rybicki, G. B. \& Hummer, D. G., 1983, ApJ, 274, 380

Smith, K. C., \& Dworetsky, M. M., 1988, in Elemental Abundance Analyses, ed. S. J. Adelman \& T. Lanz (Lausanne: Inst. Astron. Univ. Lausanne), 32

Vinkó, J., Hegedüs, T. \& Hendry, D., 1996, MNRAS, 280, 489

Wade, R. A. \& Hubeny, I., 1998, ApJ, 509, 350

Wheeler, J. C., 2007, Cosmic Catastrosphes, (Cambridge: Cambridge University Press)

Wilson, R. E., 1979, ApJ, 234, 1054

Wilson, R. E., 1990, ApJ, 356, 613

Wilson, R. E. \& Devinney, E. J., 1971, ApJ, 166, 605

Zhang, E. H., Robinson, E. L. \& Nather, R. E., 1986, ApJ, 305, 740

Zucker, S. \& Mazeh, T., 1994, ApJ, 420, 806 